\title{
Composition and Toxicity of Biogas Produced from Different Feedstocks in
}

\section{California}

Yin Li ${ }^{a}$, Christopher P. Alaimo ${ }^{a}$, Minji Kim ${ }^{a}$, Norman Y. Kado ${ }^{c}$, Joshua Peppers ${ }^{b}$, Jian Xue ${ }^{a}$, Chao Wan ${ }^{a}$, Peter G. Green ${ }^{a}$, Ruihong Zhang ${ }^{b}$, Bryan M. Jenkins ${ }^{b}$, Christoph F.A. Vogel ${ }^{c}$, Stefan Wuertz ${ }^{d}$, Thomas M. Young ${ }^{a}$, and Michael J. Kleeman ${ }^{a, *}$

${ }^{\text {a }}$ Department of Civil and Environmental Engineering, University of California - Davis, Davis, California 95616, USA

${ }^{\mathrm{b}}$ Department of Biological and Agricultural Engineering, University of California - Davis, Davis, California 95616, USA

${ }^{\mathrm{c}}$ Department of Environmental Toxicology and Center for Health and the Environment, University of California - Davis,

Davis, California 95616, USA

d Singapore Center for Environmental Life Sciences Engineering, Nanyang Technical University, Singapore 637551

*corresponding author: mjkleeman@ucdavis.edu

Department of Civil and Environmental Engineering

University of California

Davis, CA 95616

Phone: 5307528386

\begin{tabular}{|l|l|r|}
\hline Table S1 & Tedlar bag sample analysis specifications & Page S2 \\
\hline Table S2 & Target compounds in Tedlar sample bag analysis & Page S3 \\
\hline Table S3 & Sorbent tubes sampling and analysis specifications & Page S4 \\
\hline Table S4 & Target compounds and their LOQs in XAD-2 sorbent tube analysis & Page S5 \\
\hline Table S5 & Target compounds and their LOQs in charcoal sorbent tube analysis & Page S6 \\
\hline Table S6 & Target compounds and their LOQs in silica sorbent tube analysis \\
\hline Table S7 & $\begin{array}{l}\text { Concentration range of selected halocarbons }\left(\mathrm{mg} \cdot \mathrm{m}^{-3}\right) \text { measured at different landfill sites } \\
\text { and permissible occupational exposure levels by Cal/OSHA and OEHHA }\end{array}$ & Page S7 \\
\hline Table S8 & $\begin{array}{l}\text { Average concentration of BTEX measured in different biogas streams }\left(\mathrm{mg} \cdot \mathrm{m}^{-3}\right) \\
\text { permissible occupational exposure levels by Cal/OSHA and OEHHA }\end{array}$ & Page S7 \\
\hline Table S9 & Concentration of siloxanes in different biogas streams $\left(\mathrm{mg} \cdot \mathrm{m}^{-3}\right)$ & Page S8 \\
\hline Table S10 & Concentration of metals in different biogas streams $\left(\mu \mathrm{g} \cdot \mathrm{m}^{-3}\right)$ & Page S9 \\
\hline Figure S1 & Map locations of the biogas facilities studied & Page S10 \\
\hline Figure S2 & Concentration $\left(\mathrm{ppm}_{\mathrm{v}}\right.$ ) of carbonyls in different biogas streams & Page S11 \\
\hline & References & \\
\hline
\end{tabular}


Table S1 Tedlar bag sample analysis specifications

\begin{tabular}{|c|c|c|}
\hline Compounds targeted & \multicolumn{2}{|c|}{ Analysis method } \\
\hline $\begin{array}{l}\text { Major compounds } \\
\text { (carbon dioxide, methane, nitrogen, } \\
\text { oxygen, etc...) }\end{array}$ & $\begin{array}{l}\text { Agilent } 6850 \text { gas chromatograph }+ \text { thermal } \\
\text { conductivity detector }\end{array}$ & $\begin{array}{l}\text { Sample loop: } 250 \mu \mathrm{l} \text {, split ratio } 20: 1 \\
\text { Inlet temperature and pressure: } 270^{\circ} \mathrm{C}, 16 \mathrm{psi} \\
\text { Column: Agilent J\&W CP-Sil5 CB for formaldehyde } \\
60 \mathrm{mx} 0.32 \mathrm{~mm} \times 8.00 \mu \mathrm{m} \\
\text { Column flow rate: } 2.4 \mathrm{ml} / \mathrm{min} \\
\text { Total helium flow rate: } 53.7 \mathrm{ml} / \mathrm{min} \\
\text { Temperature program: }-20^{\circ} \mathrm{C} \text { for } 5 \mathrm{~min} \text {, ramp to } 150^{\circ} \mathrm{C} \text { at } \\
10^{\circ} \mathrm{C} / \mathrm{min} \text {, hold at } 150^{\circ} \mathrm{C} \text { for } 2 \mathrm{~min} \text {, ramp to } 280^{\circ} \mathrm{C} \text { at } \\
150^{\circ} \mathrm{C} / \mathrm{min} \text {, and hold for } 2 \mathrm{~min} \\
\text { Detector temperature and flow: } 250^{\circ} \mathrm{C} \text {, reference flow } \\
20 \mathrm{ml} / \mathrm{min} \text {, make-up flow } 4.6 \mathrm{ml} / \mathrm{min}\end{array}$ \\
\hline Hydrogen sulfide & $\begin{array}{l}\text { Agilent } 6850 \text { gas chromatograph }+ \text { flame } \\
\text { photometric detector }\end{array}$ & $\begin{array}{l}\text { Sample loop: } 0.1 \mathrm{ml} \text { or } 1 \mathrm{ml} \text {, split-less } \\
\text { Inlet temperature and pressure: } 50^{\circ} \mathrm{C}, 10.4 \mathrm{psi} \\
\text { Column flow rate: } 2.4 \mathrm{ml} / \mathrm{min} \\
\text { Total helium flow rate: } 53.3 \mathrm{ml} / \mathrm{min} \\
\text { Column: Agilent } \mathrm{J} \& \mathrm{~W} \mathrm{HP} 130 \mathrm{mx} 0.32 \mathrm{mmx} 5.00 \mu \mathrm{m} \\
\text { Temperature program: } 35^{\circ} \mathrm{C} \text { for } 3 \mathrm{~min} \text {, ramp to } 260^{\circ} \mathrm{C} \text { at } \\
50^{\circ} \mathrm{C} / \mathrm{min} \text {, hold at } 260^{\circ} \mathrm{C} \text { for } 4 \mathrm{~min} \\
\text { Detector temperature and flow: } 250^{\circ} \mathrm{C}, \mathrm{H}_{2} \text { flow } 50 \mathrm{ml} / \mathrm{min} \text {, air } \\
\text { flow } 60 \mathrm{ml} / \mathrm{min} \text {, make-up flow }\left(\mathrm{N}_{2}\right) 57.6 \mathrm{ml} / \mathrm{min}\end{array}$ \\
\hline $\begin{array}{l}\text { Volatile organic compounds, volatile } \\
\text { sulfur compounds (exclude } \mathrm{H}_{2} \mathrm{~S} \text { ), } \\
\text { volatile halocarbons }\end{array}$ & $\begin{array}{l}\text { Markes Unity } 2 \text { gas sampling/thermal desorption } \\
\text { system + Agilent 6890/5973N Gas chromatograph - } \\
\text { Mass spectrometer }\end{array}$ & $\begin{array}{l}\text { Sulfur trap: Markes U-T6SUL-2S, hold at } 25^{\circ} \mathrm{C} \\
\text { Sample flow: } 50 \mathrm{ml} / \mathrm{min} \text { for } 2.0 \mathrm{~min} \\
\text { Desorption: } 300^{\circ} \mathrm{C} \text { for } 3.0 \mathrm{~min} \\
\text { Transfer line: } 140^{\circ} \mathrm{C} \\
\text { Column: Agilent } \mathrm{J} \& \mathrm{~W} \mathrm{DB}-\mathrm{VRX} \text { column } \\
60 \mathrm{~m} \times 0.25 \mathrm{~mm} \times 1.40 \mu \mathrm{m} \\
\text { Temperature program: } 45^{\circ} \mathrm{C} \text { for } 3 \mathrm{~min} \text {, ramp to } 190^{\circ} \mathrm{C} \text { at } \\
10^{\circ} \mathrm{C} / \mathrm{min} \text {, ramp to } 250^{\circ} \mathrm{C} \text { at } 20^{\circ} \mathrm{C} / \mathrm{min} \text {, hold at } 250^{\circ} \mathrm{C} \text { for } 8 \mathrm{~min}\end{array}$ \\
\hline
\end{tabular}


Table S2 Target compounds in Tedlar sample bag analysis

\begin{tabular}{|c|c|c|c|c|}
\hline Major compounds & Volatile sulfur compounds & \multicolumn{2}{|c|}{ Volatile halocarbons } & \multirow{2}{*}{$\begin{array}{c}\begin{array}{c}\text { Volatile organic } \\
\text { compounds }\end{array} \\
\text { 1,3-Butadiene }\end{array}$} \\
\hline Nitrogen/Carbon Monoxide & Sulfur Dioxide & Dichlorodifluoromethane & 1,1,2,3,4,4-hexachloro-1,3- & \\
\hline Oxygen/Argon & Carbonyl sulfide & 1,2-dichloro-1,1,2,2-tetrafluoroethane & Butadiene & \\
\hline Methane & Carbon disulfide & 1,1,2-trichloro-1,2,2-trifluoroethane & Chlorobenzene & Toluene \\
\hline Carbon Dioxide & Methyl mercaptan & Trichlorofluoromethane & 1,2-dichlorobenzene & Ethylbenzene \\
\hline Ammonia & Ethyl mercaptan & Dichloromethane & 1,3-dichlorobenzene & m,p-Xylene \\
\hline Hydrogen & Isopropyl mercaptan & Chloroform & 1,4-Dichlorobenzene & o-Xylene \\
\hline Ethane & n-Propyl mercaptan & Carbon Tetrachloride & 1,2,3-Trichlorobenzene & Styrene \\
\hline Ethene & t-Butyl mercaptan & Chloroethane & 1,2,4-trichlorobenzene & Isopropylbenzene \\
\hline Ethyne & Dimethyl sulfide & 1,1-dichloroethane & 2-Chlorotoluene & 4-Ethyltoluene \\
\hline Propane & Methyl Ethyl sulfide & 1,2-Dichloroethane & 4-Chlorotoluene & n-Propylbenzene \\
\hline Propene & Diethyl sulfide & 1,1,1-trichloroethane & Bromomethane & 1,3,5-trimethylbenzene \\
\hline Propadiene & Di-tert-butyl sulfide & 1,1,2-trichloroethane & dibromomethane & tert-butylbenzene \\
\hline Propyne & Dimethyl Disulfide & 1,1,1,2-tetrachloroethane & Bromoform & 1,2,4-Trimethylbenzene \\
\hline i-Butane & Diethyl Disulfide & 1,1,2,2-tetrachloroethane & bromochloromethane & s-Butylbenzene \\
\hline n-Butane & Methyl Ethyl Disulfide & Chloroethene & bromodichloromethane & p-Isopropyltoluene \\
\hline 1-Butene & Methyl i-Propyl Disulfide & 1,1-dichloroethene & dibromochloromethane & n-butylbenzene \\
\hline i-Butene & Methyl n-Propyl Disulfide & cis-1,2-Dichloroethene & 1,2-dibromoethane & Naphthalene \\
\hline trans-2-Butene & Methyl t-Butyl Disulfide & Trans-1,2-dichloroethene & Bromochloroethane & Pyridine \\
\hline cis-2-Butene & Ethyl i-Propyl Disulfide & Trichloroethene & 1,2-dibromo-3- & Nitrobenzene \\
\hline 1,3-Butadiene & Ethyl n-Propyl Disulfide & Tetrachloroethene & chloropropane & \\
\hline Isoprene & Ethyl t-Butyl Disulfide & 1,2-dichloropropane & bromobenzene & \\
\hline i-Pentane & Di-i-Propyl Disulfide & 2,2-dichloropropane & & \\
\hline n-Pentane & i-Propyl n-Propyl Disulfide & 1,2,3-trichloropropane & & \\
\hline neo-Pentane & Di-n-Propyl Disulfide & 3-chloropropene & & \\
\hline Pentenes & i-Propyl t-Butyl Disulfide & 1,1-dichloropropene & & \\
\hline & n-Propyl t-Butyl Disulfide & cis-1,3-dichloropropene & & \\
\hline & Di-t-Butyl Disulfide & trans-1,3-dichloropropene & & \\
\hline
\end{tabular}


Table S3 Sorbent tubes sampling and analysis specifications

\begin{tabular}{|c|c|c|c|c|c|}
\hline Sampling media & Compounds targeted & $\begin{array}{l}\text { Sampling } \\
\text { duration }\end{array}$ & $\begin{array}{c}\text { Extraction } \\
\text { method }\end{array}$ & \multicolumn{2}{|c|}{ Analysis method } \\
\hline Charcoal tube & $\begin{array}{c}\text { Extended hydrocarbons, } \\
\text { Siloxanes }\end{array}$ & $\begin{array}{l}60 \mathrm{~min} \text { at } \\
1 \mathrm{~L} \mathrm{~min}^{-1}\end{array}$ & $\begin{array}{l}\text { 1ml ethyl acetate } \\
\text { Sonicate } 30 \mathrm{~min}\end{array}$ & $\begin{array}{c}\text { Agilent } 7890 \text { gas } \\
\text { chromatograph } \\
+ \\
\text { Agilent } 7200 \text { quadrupole } \\
\text { time-of-flight mass } \\
\text { spectrometer }\end{array}$ & $\begin{array}{l}\text { Injection volume: } 1.0 \mu \mathrm{l} \\
\text { Injection temperature: } 250^{\circ} \mathrm{C} \\
\text { Column: Agilent } \mathrm{J} \& \mathrm{~W} \text { HP5-MS UI } \\
\text { 30mx0.25mmx0.25 } \mathrm{mm} \\
\text { Column flow: Helium } 0.8 \mathrm{ml} / \mathrm{min} \\
\text { Temperature program: } 35^{\circ} \mathrm{C} \text { for } \\
3 \mathrm{~min}, 30^{\circ} \mathrm{C} \text { to } 325^{\circ} \mathrm{C} \text { at } 4^{\circ} \mathrm{C} / \mathrm{min} \text {, } \\
\text { hold at } 325^{\circ} \mathrm{C} \text { for } 3 \mathrm{~min}\end{array}$ \\
\hline XAD-2 tube & $\begin{array}{c}\text { Semi-volatile organic } \\
\text { compounds, } \\
\text { Polycyclic aromatic } \\
\text { hydrocarbons, } \\
\text { Polychlorinated biphenyls, } \\
\text { Semi-volatile sulfur compounds, } \\
\text { Pesticides }\end{array}$ & $\begin{array}{l}60 \mathrm{~min} \text { at } \\
1 \mathrm{~L} \mathrm{~min}^{-1}\end{array}$ & $\begin{array}{l}\text { 1ml ethyl acetate } \\
\text { Sonicate } 30 \mathrm{~min}\end{array}$ & $\begin{array}{c}\text { Agilent } 7890 \text { gas } \\
\text { chromatograph } \\
+ \\
\text { Agilent } 7200 \text { quadrupole } \\
\text { time-of-flight mass } \\
\text { spectrometer }\end{array}$ & $\begin{array}{l}\text { Injection volume: } 1.0 \mu \mathrm{l} \\
\text { Injection temperature: } 250^{\circ} \mathrm{C} \\
\text { Column: Agilent } \mathrm{J} \& \mathrm{~W} \text { HP5-MS UI } \\
\text { 30mx0.25mmx0.25 } \mathrm{mm} \\
\text { Column flow: Helium } 0.8 \mathrm{ml} / \mathrm{min} \\
\text { Temperature program: } 35^{\circ} \mathrm{C} \text { for } \\
3 \mathrm{~min}, 30^{\circ} \mathrm{C} \text { to } 325^{\circ} \mathrm{C} \text { at } 4^{\circ} \mathrm{C} / \mathrm{min} \text {, } \\
\text { hold at } 325^{\circ} \mathrm{C} \text { for } 3 \mathrm{~min}\end{array}$ \\
\hline $\begin{array}{c}\text { Silica tube with } \\
\text { dinitrophenylhydrazine } \\
\text { (DNPH) coating }\end{array}$ & Carbonyls & $\begin{array}{l}1 \mathrm{~min} \text { at } \\
1 \mathrm{~L} \mathrm{~min}^{-1}\end{array}$ & $\begin{array}{l}\text { 1ml acetonitrile } \\
\text { Sonicate } 30 \mathrm{~min}\end{array}$ & $\begin{array}{c}\text { Agilent } 1200 \text { liquid } \\
\text { chromatograph } \\
+ \\
\text { Agilent } 6530 \text { quadrupole } \\
\text { time-of-flight mass } \\
\text { spectrometer }\end{array}$ & $\begin{array}{l}\text { Injection volume: } 10 \mu \mathrm{l} \\
\text { Column: Restek Ultra } \mathrm{C}_{18} 5 \mu \mathrm{m} \\
\text { 250x4.6mm } \\
\text { Gradient program: } 40 \% \mathrm{~A} \\
\text { (deionized water with } 1 \mathrm{mM} \\
\text { ammonium acetate) and } 60 \% \mathrm{~B} \\
\text { (acetonitrile/ } \mathrm{H}_{2} \mathrm{O}, 95 / 5 \mathrm{v} / \mathrm{v} \text {, with } \\
1 \mathrm{mM} \text { ammonium acetate) for } 7 \mathrm{~min} \text {, } \\
\text { linear increase to } 100 \% \mathrm{~B} \text { at } 20 \mathrm{~min} \text {, } \\
\text { hold at } 100 \% \text { B for } 0.5 \mathrm{~min}\end{array}$ \\
\hline
\end{tabular}


Table S4 Target compounds and their LOQs in XAD-2 sorbent tube analysis

\begin{tabular}{|c|c|c|c|c|c|c|c|}
\hline $\begin{array}{l}\text { Semi-volatile sulfur- } \\
\text { containing compounds }\end{array}$ & $\begin{array}{l}\text { LOQ } \\
\text { (ppbv) }\end{array}$ & $\begin{array}{l}\text { Semi-volatile organic } \\
\text { compounds and PAHs }\end{array}$ & $\begin{array}{c}\text { LOQ } \\
\text { (ppbv) }\end{array}$ & $\begin{array}{l}\text { Semi-volatile organic } \\
\text { compounds and PAHs }\end{array}$ & $\begin{array}{c}\text { LOQ } \\
\text { (ppbv) }\end{array}$ & Pesticides & $\begin{array}{l}\text { LOQ } \\
\text { (ppbv) }\end{array}$ \\
\hline Dimethyl Trisulfide & 0.007 & 2,4-dichlorophenol & 0.057 & 4-chlorophenyl phenyl ether & 0.009 & a-BHC & 0.006 \\
\hline Diethyl Trisulfide & 0.007 & 4-Chloroaniline & 0.029 & 4-Nitroaniline & 0.135 & b-BHC & 0.013 \\
\hline Di-t-Butyl Trisulfide & 0.007 & 4-chloro-3-methylphenol & 0.003 & Diphenylamine & 0.011 & g-BHC & 0.013 \\
\hline Thiophene & 10 & 2-methylnaphthalene & 0.013 & Azobenzene & 0.020 & d-BHC & 0.006 \\
\hline C1-Thiophenes & 10 & 1-methylnaphthalene & 0.003 & 4-Bromophenyl phenyl ether & 0.007 & Heptachlor & 0.005 \\
\hline C2-Thiophenes & 0.017 & Hexachlorocyclopentadiene & 0.014 & Hexachlorobenzene & 0.007 & Aldrin & 0.001 \\
\hline C3-Thiophenes & 0.007 & 2,4,6-trichlorophenol & 1.890 & Pentachlorophenol & 1.401 & Heptachlor epoxide & 0.002 \\
\hline Benzothiophene & 0.007 & 2,4,5-trichlorophenol & 0.472 & Phenanthrene & 0.021 & g-Chlordane & 0.001 \\
\hline C1-Benzothiophenes & 0.063 & 2-chloronaphthalene & 0.006 & Anthracene & 0.052 & Endosulfan I & 0.005 \\
\hline C2-Benzothiophenes & 0.006 & 2-Nitroaniline & 0.068 & Carbazole & 0.056 & a-Chlordane & 0.002 \\
\hline Thiophane & 10 & 1,4-dinitrobenzene & 0.222 & Di-n-butyl phthalate & 0.013 & Dieldrin & 0.010 \\
\hline Thiophenol & 10 & Dimethyl phthalate & 0.048 & Fluoranthene & 0.005 & 4,4'-DDE & 0.006 \\
\hline $\begin{array}{l}\text { Semi-volatile organic } \\
\text { compounds and PAHs }\end{array}$ & $\begin{array}{l}\text { LOQ } \\
\text { (ppbv) }\end{array}$ & 1,3-dinitrobenzene & 0.222 & Pyrene & 0.002 & Endrin & 0.010 \\
\hline N-nitrosodimethylamine & 1.259 & 2,6-dinitrotoluene & 0.102 & Benzyl butyl phthalate & 0.060 & Endosulfan II & 0.009 \\
\hline Phenol & 5.000 & Acenaphthylene & 0.002 & Bis(2-ethylhexyl)adipate & 0.025 & 4,4'-DDD & 0.006 \\
\hline 2-Chlorophenol & 0.007 & 1,2-Dinitrobenzene & 0.555 & Benzo(a)anthracene & 0.008 & Endrin aldehyde & 0.024 \\
\hline 2-methylphenol & 0.017 & 3-Nitroaniline & 0.068 & Chrysene & 0.008 & Endosulfan sulfate & 0.002 \\
\hline bis(2-chloroisopropyl)ether & 0.055 & Acenaphthene & 0.006 & Bis(2-ethylhexyl)phthalate & 0.048 & 4,4'-DDT & 0.005 \\
\hline N-Nitroso-di-n-propylamine & 0.029 & 4-nitrophenoI & 0.671 & Di-n-octyl phthalate & 0.048 & Endrin ketone & 0.010 \\
\hline 3-methylphenol & 0.017 & Dibenzofuran & 0.011 & Benzo(b)fluoranthene & 0.037 & Methoxychlor & 0.011 \\
\hline 4-methylphenol & 0.003 & 2,4-dinitrotoluene & 0.102 & Benzo(k)fluoranthene & 0.037 & & \\
\hline lsophorone & 0.027 & 2,3,4,6-Tetrachlorophenol & 0.402 & Benzo(a)pyrene & 0.037 & & \\
\hline 2-nitrophenoI & 0.013 & 2,3,5,6-Tetrachlorophenol & 1.609 & Indeno(1,2, 3-cd)pyrene & 0.135 & & \\
\hline 2,4-dimethylphenol & 0.031 & Diethyl Phthalate & 0.084 & Dibenzo(a, h)anthracene & 0.134 & & \\
\hline Bis(2-chloroethoxy)methane & 0.011 & Fluorene & 0.022 & Benzo[g,h,i)perylene & 0.068 & & \\
\hline
\end{tabular}


Table S5 Target compounds and their LOQs in charcoal sorbent tube analysis

\begin{tabular}{|c|c|c|c|c|c|}
\hline Organic silicon compounds & LOQ (ppbv) & Extended hydrocarbons & LOQ (ppbv) & Extended hydrocarbons & LOQ (ppbv) \\
\hline 1,1,3,3-Tetramethyldisiloxane & 5 & Cyclopentane & 1.875 & Nonanes & 0.029 \\
\hline Pentamethyldisiloxane & 5 & Methylcyclopentane & 1.875 & Decanes & 0.003 \\
\hline Hexamethyldisilane & 5 & Cyclohexane & 1.875 & Undecanes & 0.012 \\
\hline Hexamethyldisiloxane & 5 & Methylcyclohexane & 1.870 & Dodecanes & 0.002 \\
\hline Octamethyltrisiloxane & 0.04 & C3 Benzenes & 0.008 & Tridecanes & 0.002 \\
\hline Octamethylcyclotetrasiloxane & 0.03 & C1 Naphthalenes & 0.007 & Tetradecanes & 0.009 \\
\hline Decamethyltetrasiloxane & 0.03 & C2 Naphthalenes & 0.012 & Pentadecanes & 0.009 \\
\hline Decamethylcyclopentasiloxane & 0.03 & Hexanes & 1.875 & Hexadecanes & 0.002 \\
\hline Dodecamethylpentasiloxane & 0.02 & Heptanes & 1.901 & Heptadecanes & 0.002 \\
\hline \multirow[t]{3}{*}{ Dodecamethylcyclohexasiloxane } & 0.04 & 2,2,4-Trimethylpentane & 1.870 & Octadecanes & 0.004 \\
\hline & & Octanes & 1.870 & Nonadecanes & 0.004 \\
\hline & & & & Eicosanes+ & 0.003 \\
\hline
\end{tabular}

Table S6 Target compounds and their LOQs in silica sorbent tube analysis

\begin{tabular}{|c|c|c|}
\hline Carbonyl compounds & LOQ (ppbv) & Carbonyl compounds \\
\hline Formaldehyde & 0.006 & Methacrolein (lsobutenal) \\
\hline Acetaldehyde & 0.001 & Butyraldehyde (Butanal) \\
\hline Acrolein (2-propenal) & 0.003 & Benzaldehyde \\
\hline Propionaldehyde & 0.003 & Valeraldehyde (Pentanal) \\
\hline Crotonaldehyde & 0.053 & p-Tolualdehyde \\
\hline 2-Butanone (MEK) & 0.026 & Hexanaldehyde (Hexanal) \\
\hline
\end{tabular}


Table S7 Concentration range of selected halocarbons $\left(\mathrm{mg} \cdot \mathrm{m}^{-3}\right)$ measured at different landfill sites and permissible occupational exposure levels by Cal/OSHA and OEHHA

\begin{tabular}{|c|c|c|c|c|c|}
\hline & $\begin{array}{c}\text { Landfill biogas (5\&6) } \\
\text { in this study }\end{array}$ & $\begin{array}{l}\text { Eklund et al. } \\
\qquad(1998)^{1}\end{array}$ & $\begin{array}{c}\text { Allen et al. } \\
(1997)^{2}\end{array}$ & $\begin{array}{c}\text { Cal/OSAH } \\
\text { PELs }^{3} \\
\end{array}$ & OEHHA $^{4}$ \\
\hline Total Halocarbons & $9.90-15.65$ & - & 246-1239 & - & - \\
\hline Dichlorodifluromethane & ND*-1.68 & 6.28 & $<0.5-231$ & 4950 & - \\
\hline Trichlorofluromethane & ND-0.21 & - & $<0.5-74$ & 5600 & - \\
\hline Chloroethene & ND-0.24 & - & $<0.1-87$ & - & 0.84 \\
\hline 1,2-Dichloroethene & ND-1.99 & - & $1-182$ & 790 & - \\
\hline Trichloroethene & ND-0.48 & - & $<0.1-152$ & 135 & - \\
\hline Tetrachloroethene & ND-1.10 & - & $<0.1-255$ & 170 & - \\
\hline Chlorobenzene & ND-0.74 & 5.29 & - & 46 & - \\
\hline Dichlorobenzene & ND-3.99 & 24.35 & - & 60 & 5.7 \\
\hline
\end{tabular}

${ }^{*}$ ND means not detected

Table S8 Average concentration of BTEX (Benzene, Toluene, Ethylbenzene and Xylenes) measured in different biogas streams $\left(\mathrm{mg} \cdot \mathrm{m}^{-3}\right)$ and permissible occupational exposure levels by Cal/OSHA and OEHHA

\begin{tabular}{ccccc}
\hline & Benzene & Toluene & Ethylbenzene & Xylene \\
\cline { 2 - 5 } Biogas 1 & 0.04 & 0.15 & 0.10 & 0.27 \\
Biogas 2 & 0.56 & 0.73 & 0.06 & 0.20 \\
Biogas 3 & 0.22 & 0.37 & 0.27 & 0.09 \\
Biogas 4 & 0.27 & 3.15 & 2.20 & 3.55 \\
Biogas 5 & 1.76 & 5.46 & 3.95 & 8.06 \\
Biogas 6 $^{\text {Cal/OSHA PELs }}$ (OSHA $^{\mathbf{3}}$ & 3.04 & 5.79 & 4.16 & 9.63 \\
OEHHA4 $^{4}$ & $\mathbf{3 . 1 9}$ & $\mathbf{3 7}$ & $\mathbf{2 2}$ & $\mathbf{4 3 5}$ \\
\hline
\end{tabular}


Table S9 Concentration of siloxanes in different biogas streams $\left(\mathrm{mg} \cdot \mathrm{m}^{-3}\right)$

\begin{tabular}{|c|c|c|c|c|c|c|c|c|}
\hline & L2 & D3 & L3 & D4 & L4 & D5 & L5 & D6 \\
\hline Biogas 4 & 9.996 & - & 0.049 & 0.001 & 0.008 & 1.258 & 0.002 & 0.031 \\
\hline Biogas 5 & 2.253 & - & 0.086 & 0.000 & 0.050 & 0.000 & 0.001 & 0.002 \\
\hline Biogas 6 & 2.858 & - & 0.004 & 0.125 & 0.000 & 0.018 & ND & ND \\
\hline (Urban et al., 2009) $^{5}$ & $3.1-5.0$ & $0.5-0.84$ & - & $10.6-15$ & $<0.1$ & 3.0-3.3 & - & - \\
\hline$(\text { Rasi, 2009) })^{6}$ & $0.03-0.63$ & $<0.1$ & $<0.01$ & $<0.67$ & ND & $<0.3$ & - & - \\
\hline $\begin{array}{l}\text { (Schweigkofler and } \\
\text { Niessner, 2001) }\end{array}$ & $0.7-0.9$ & $0.4-0.44$ & - & $4.8-5.1$ & - & $0.6-0.65$ & - & - \\
\hline (Schweigkofler and & $1.04-1.31$ & 0.01 & $0.03-0.05$ & 7.97-8.84 & $<0.01$ & $0.5-1.09$ & - & ND \\
\hline Niessner, 1999) ${ }^{8}$ & $0.38-0.77$ & $0.31-0.45$ & 0.04 & $4.24-5.03$ & $<0.01$ & $0.4-0.53$ & - & ND \\
\hline
\end{tabular}


Table S10 Concentration of metals in different biogas streams. Results expressed in (average value \pm 1 standard deviation). Concentrations below limit of detection (LOD) are denoted as $0 \pm 0$. All values in unit $\mu \mathrm{g} \cdot \mathrm{m}^{-3}$

\begin{tabular}{|c|c|c|c|c|c|c|c|}
\hline Element & LOD & Biogas 1 & Biogas 2 & Biogas 3 & Biogas 4 & Biogas 5 & Biogas 6 \\
\hline Be & 0.005 & $0 \pm 0$ & $0 \pm 0$ & $0.009 \pm 0.009$ & $0 \pm 0$ & $0.013 \pm 0.004$ & $0 \pm 0$ \\
\hline $\mathrm{Cr}$ & 0.005 & $0.926 \pm 1.31$ & $0.061 \pm 0.028$ & $0.21 \pm 0.24$ & $0.34 \pm 0.76$ & $0 \pm 0$ & $0.19 \pm 0.14$ \\
\hline Mn & 0.005 & $0 \pm 0$ & $0.067 \pm 0.047$ & $0.005 \pm 0.008$ & $0 . .48 \pm 0.95$ & $0 \pm 0$ & $0.24 \pm 0.26$ \\
\hline Co & 0.005 & $0.00617 \pm 0.00873$ & $0.006 \pm 0.008$ & $0.018 \pm 0.013$ & $0.062 \pm 0.14$ & $0 \pm 0$ & $0.003 \pm 0.005$ \\
\hline $\mathrm{Ni}$ & 0.02 & $0.272 \pm 0.384$ & $0.12 \pm 0.15$ & $0.074 \pm 0.13$ & $0 \pm 0$ & $0 \pm 0$ & $0 \pm 0$ \\
\hline $\mathrm{Zn}$ & 0.2 & $0 \pm 0$ & $7.8 \pm 8.7$ & $0.56 \pm 0.96$ & $0.14 \pm 0.3$ & $0 \pm 0$ & $0.56 \pm 0.96$ \\
\hline Se & 0.2 & $0.648 \pm 0.458$ & $0.21 \pm 0.30$ & $0.14 \pm 0.24$ & $0.45 \pm 0.65$ & $0.15 \pm 0.15$ & $0.20 \pm 0.35$ \\
\hline $\mathrm{Sr}$ & 0.01 & $0 \pm 0$ & $0.005 \pm 0.008$ & $0 \pm 0$ & $0 \pm 0$ & $0.1 \pm 0.1$ & $0.009 \pm 0.016$ \\
\hline Mo & 0.005 & $0.0988 \pm 0.114$ & $14.1 \pm 20.4$ & $0.009 \pm 0.009$ & $0 \pm 0$ & $0 \pm 0$ & $0 \pm 0$ \\
\hline $\mathrm{Cd}$ & 0.005 & $0.204 \pm 0.249$ & $0 \pm 0$ & $0.005 \pm 0.008$ & $0.003 \pm 0.007$ & $0 \pm 0$ & $0 \pm 0$ \\
\hline $\mathrm{Ba}$ & 0.02 & $0.0247 \pm 0.0349$ & $0 \pm 0$ & $0 \pm 0$ & $1.6 \pm 2.2$ & $1.5 \pm 1.5$ & $0 \pm 0$ \\
\hline $\mathrm{Hg}$ & 0.005 & $0 \pm 0$ & $0 \pm 0$ & $0 \pm 0$ & $0.006 \pm 0.014$ & $0.008 \pm 0.008$ & $0 \pm 0$ \\
\hline $\mathrm{Tl}$ & 0.005 & $0.00617 \pm 0.00873$ & $0.011 \pm 0.016$ & $0.014 \pm 0.015$ & $0 \pm 0$ & $0 \pm 0$ & $0.003 \pm 0.005$ \\
\hline $\mathrm{Cu}$ & 0.005 & $0 \pm 0$ & $0.006 \pm 0.008$ & $0.005 \pm 0.008$ & $0 \pm 0$ & $0 \pm 0$ & $0.20 \pm 0.34$ \\
\hline As & 0.005 & $0.315 \pm 0.432$ & $0.022 \pm 0.021$ & $0.23 \pm 0.38$ & $1.6 \pm 1.4$ & $8.5 \pm 3.4$ & $4.2 \pm 2.3$ \\
\hline $\mathrm{Sb}$ & 0.005 & $0.259 \pm 0.184$ & $0.028 \pm 0.028$ & $0.31 \pm 0.17$ & $1.6 \pm 1.8$ & $12.5 \pm 12.5$ & $1.3 \pm 2.0$ \\
\hline $\mathrm{Pb}$ & 0.1 & $1.73 \pm 2.44$ & $0 \pm 0$ & $0 \pm 0$ & $0.14 \pm 0.31$ & $0.8 \pm 0.8$ & $0.65 \pm 1.1$ \\
\hline $\mathrm{Na}$ & 2 & $0 \pm 0$ & $0 \pm 0$ & $0 \pm 0$ & $0 \pm 0$ & $0 \pm 0$ & $0 \pm 0$ \\
\hline $\mathrm{Mg}$ & 0.2 & $0 \pm 0$ & $0.56 \pm 0.79$ & $3.0 \pm 4.9$ & $0 \pm 0$ & $6.2 \pm 6.2$ & $0 \pm 0$ \\
\hline $\mathrm{Al}$ & 0.2 & $0 \pm 0$ & $0 \pm 0$ & $0 \pm 0$ & $2.2 \pm 5$ & $0 \pm 0$ & $5.6 \pm 9.6$ \\
\hline $\mathrm{K}$ & 1 & $0 \pm 0$ & $0 \pm 0$ & $1.02 \pm 1.76$ & $0 \pm 0$ & $0 \pm 0$ & $0 \pm 0$ \\
\hline $\mathrm{Ca}$ & 1 & $0 \pm 0$ & $92.6 \pm 89.7$ & $8.3 \pm 14.4$ & $1.2 \pm 2.6$ & $54 \pm 52$ & $14.5 \pm 23.7$ \\
\hline $\mathrm{Fe}$ & 1 & $1.91 \pm 2.71$ & $0 \pm 0$ & $4.4 \pm 7.6$ & $9.6 \pm 21$ & $0 \pm 0$ & $0 \pm 0$ \\
\hline Sn & 0.02 & $0 \pm 0$ & $0 \pm 0$ & $0.88 \pm 0.19$ & $0.05 \pm 0.11$ & $0.55 \pm 0.05$ & $0.40 \pm 0.56$ \\
\hline
\end{tabular}




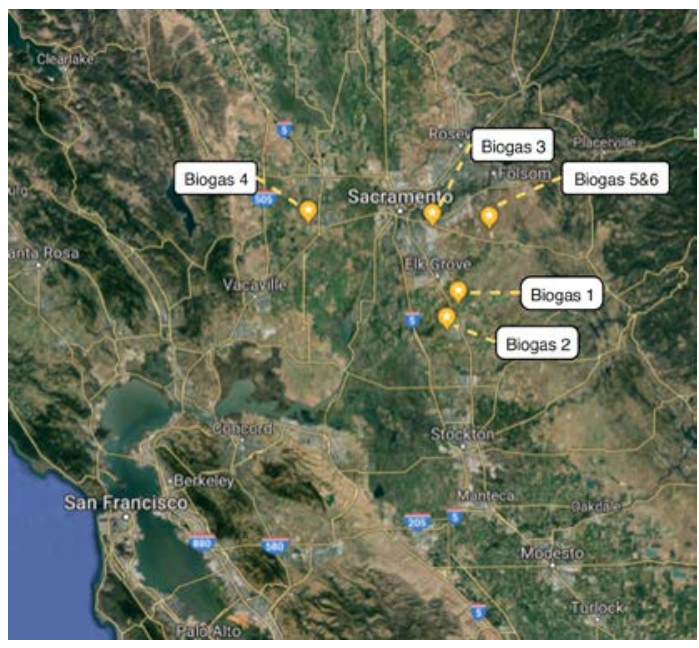

Figure S1 Map locations of the biogas facilities studied. Map from Google Maps:

https://goo.gl/maps/ogpi5QAiX4n2qhXk6

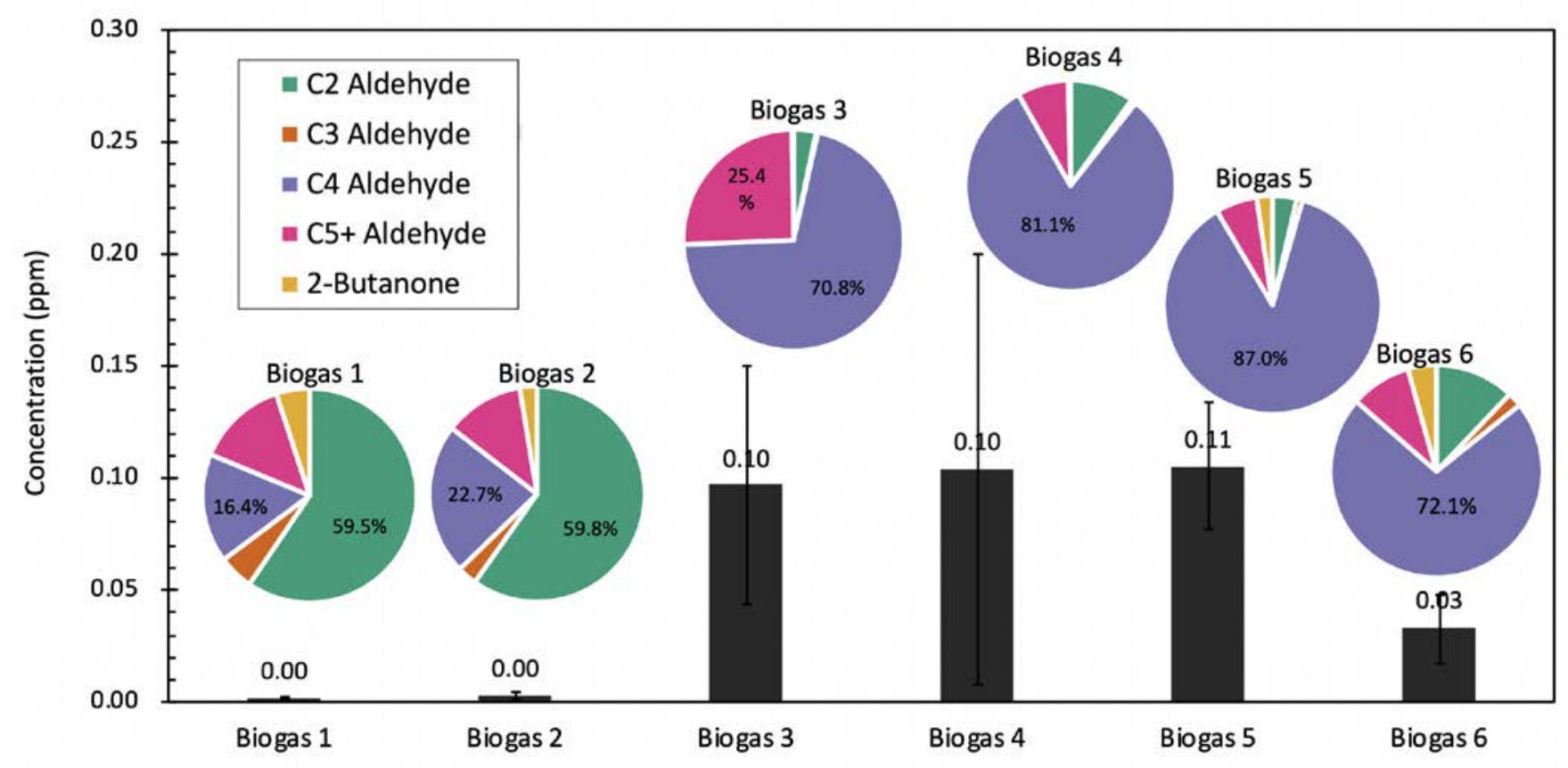

Figure S2 Concentration $\left(\mathrm{ppm}_{\mathrm{v}}\right.$ ) of carbonyls in different biogas st reams.

Error bars represent 1 standard deviation of total carbonyl concentration. Chemical species quantified include acetaldehyde (C2), propionaldehyde, acrolein (C3), butyraldehyde, methacrolein (C4), (iso)valeraldehyde, hexanaldehyde, benzaldehyde, 2,5-Dimethylbenzaldehyde (C5+), and 2-butanone. 


\section{References}

(1) Eklund, B.; Anderson, E. P.; Walker, B. L.; Burrows, D. B. Characterization of Landfill Gas Composition at the Fresh Kills Municipal Solid-Waste Landfill. Environ. Sci. Technol. 1998, 32 (15), 2233-2237. https://doi.org/10.1021/es980004s.

(2) Allen, M. R.; Braithwaite, A.; Hills, C. C. Trace Organic Compounds in Landfill Gas at Seven U.K. Waste Disposal Sites. Environ. Sci. Technol. 1997, 31 (4), 1054-1061. https://doi.org/10.1021/es9605634.

(3) Cal/OSHA. California division of occupational safety and health administration (Cal/OSHA) permissible exposure limits (PELs) for chemical contaminants Table AC-1 https://www.dir.ca.gov/title8/ac1.pdf.

(4) CalEPA. California Environmental Protection Agency; Recommendations to the California Public Utilities Commission Regarding Health Protective Standards for the Injection of Biomethane into the Common Carrier Pipeline. Available

https//www.arb.ca.gov/energy/biogas/documents/FINAL_AB_1900_Staff_Report_\&_Appendices_\%20051513.pdf. Last access August 2017 2013. https://doi.org/10.1007/s11104-007-9437-8.

(5) Urban, W.; Lohmann, H.; Gómez, J. I. S. Catalytically Upgraded Landfill Gas as a Cost-Effective Alternative for Fuel Cells. J. Power Sources 2009, 193 (1), 359-366. https://doi.org/10.1016/j.jpowsour.2008.12.029.

(6) Rasi, S. Biogas Composition and Upgrading to Biomethane, 2009. https://doi.org/978-951-39-3607-5.

(7) Schweigkofler, M.; Niessner, R. Removal of Siloxanes in Biogases. J. Hazard. Mater. 2001, 83 (3), $183-196$. https://doi.org/10.1016/S0304-3894(00)00318-6.

(8) Schweigkofler, M.; Niessner, R. Determination of Siloxanes and VOC in Landfill Gas and Sewage Gas by Canister Sampling and GC-MS/AES Analysis. Environ. Sci. Technol. 1999, 33 (20), 3680-3685. https://doi.org/10.1021/es9902569. 